\title{
Life-threatening Streptococcal Myonecrosis Complicating Primary Wound Closure after Pilonidal Sinus Excision
}

\author{
Col T A Panov \\ $M D, M C B G$ \\ Senior Surgeon \\ K T Kjossev \\ MD
}

Research Fellow

J E Losanoff

MD

Assistant Professor of Surgery

Military Medical Academy, Department of General Surgery, 3, Georgi Sofijski Blvd, 1606 Sofia, Bulgaria

SUMMARY: We describe a case of anaerobic streptococcal myonecrosis as a rare complication after pilonid搽 sinus excision with primary closure. The recognition of the disease in any patient who has early septic deterioratio $\$$ after a surgical procedure requires aggressive management for survival.

\section{Introduction}

Most reports have described the polymicrobial nature and predominance of anaerobic bacteria in infected pilonidal sinuses $(1,2)$. However, anaerobic infections as secondary complications of surgical procedures for pilonidal sinuses are uncommon entities. In general, their pathogenesis involves a complex of factors, such as the virulence of the invading organisms, the state of the host's defenses, devitalized tissue and/or a cavity creating an anaerobic environment, and foreign body. Many of these factors can be implicated after excision of a pilonidal sinus with primary closure.

The purpose of this report is to illustrate the clinical and pathological findings of anaerobic streptococcal myonecrosis following pilonidal sinus excision with primary closure. We have not come across any report in the available literature and this has prompted us to describe this case.

\section{Case report}

Six days before admission to our institution, a 19 year old female was admitted to another hospital with 18 months history of sacrococcigeal pilonidal sinus and repeated foul odour secretion. At operation $5 \times 3$ centimetres pilonidal sinus was excised with primary closure of the cavity and tube drainage. The postoperative course was uneventful for 5 days, at which time fever and pain in the wound area developed. Re-exploration revealed induration of the area and $20 \mathrm{ml}$ of foul-smelling purulent material was evacuated; the wound was left open. On the next day patient's condition deteriorated with hypotension fever and impaired consciousness. The patient was prepared for emergency wound revision, at which the subcutaneous tissue was seen to be oozing. The fascia and gluteus group were necrotic and filled with Wound debridement and resection of the necrotic mustes was performed. An anaerobic infection was suspectedọ్nd periwound area was deeply incised. In view of severity of the patient's condition which did not resip/, to an initial period of treatment, she was transferred abtle 8th postoperative day to the Military Medical Acadelng by use of helicopter service.

On admission the examination revealed a lethagge young female with signs of multi-organ-sys disfunction. Her temperature was $40^{\circ} \mathrm{C}$, pulse rate 1 . beats/minute, respiratory rate 26 breaths/minute, ar blood pressure $90 / 60 \mathrm{~mm} \mathrm{Hg}$. Her tongue was furred argi her liver palpable to two centimetres below the rig costal margin. In the area involving the natal crease from the broad triangular area over the upper part of the sacrum, down to some 2,5 to 3,0 centimeters from tow anus and buttocks, a $50 \times 23$ centimeters brown-greenis irregular wound was seen. The gluteal muscles gave sweet but foul odour and gas bubbles were visible in tim discharge. Crepitus was present in the periwound area.

The admission laboratory test revealed the followirs values: hematocrit $-0,33$, WBC count $-6,0 \times 10^{\circ} / \mathrm{L}$, blo ज्ञ़ी urea - 29,3 mmol/L, SGOT - $254 \mathrm{U} / \mathrm{L}$. SGPT - $48 \mathrm{U} / \mathrm{L}$.

Chest X-Ray was normal. Material obtained by need aspiration in the wound region revealed large gran positive cocci, which later proved to to Peptostreptococcus. In addition Staphylococcus faecalis Staphylococcus epidermidis and Proteus mirabilis we present.

A diagnosis of anaerobic streptococcal myonecrosis was made and the patient was treated accordingly. Sl: was admitted to the Intensive Care Unit where nutritiona 
support and penicillin therapy was started together with repeated wound packing and debridement.

On the 10th postoperative day the infection was noted to be extending laterally and deeply down the right sciatic nerve (Fig 1). The patient developed symptoms of classic sciatica, with pain radiating down the back of the leg aggravated by dorsiflexion and muscle weakness. Subsequently, culture specimens of pus and muscle grew the same organisms. Culture of blood grew Seratia marcesens, a fact that required further change in antibiotic therapy.

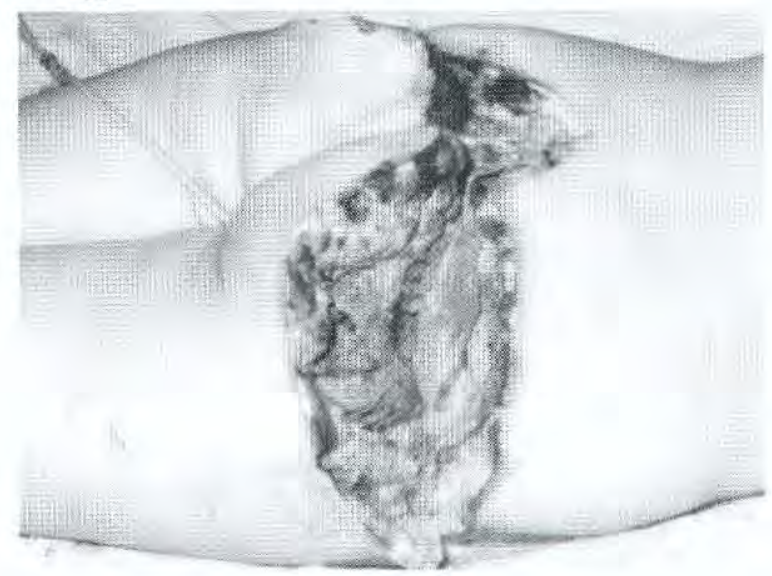

Fig 1. View on the 10th postoperative day shows extent of spread of anaerobic streptococcal myonecrosis laterally and deeply down the right sciatic nerve.

The patient was returned to the operating room on five occasions over the next several days for dressing changes with whirlpool baths and further debridement of obviously necrotic muscle. Hyperbaric oxygen therapy as an additive treatment was begun because of extensive involvement of the sciatic area where adequate debridement was difficult. Two weeks later good granulation tissue was present. After excision, the wound was sutured and partially closed. A skin defect remained of $100 \mathrm{~cm}^{2}$ at the left and of $200 \mathrm{~cm}^{2}$ on the right. One month later the second stage of plastic reconstruction was performed. The postoperative course was uneventful and patient was discharged 79 days after the initial operation.

At follow-up 12 months later, the patient was entirely free of local and neurologic symptoms, and was 32 weeks pregnant.

\section{Discussion}

Anaerobic streptococcal myonecrosis is a rare condition, usually accompanied by severe systemic toxicity and easily mistaken for clostridial gas gangrene. The correct diagnosis is reached after the recovery of peptostreptococci of anaerobic culture. Typically, infection followed deep penetrating wounds, but sometimes involvement of the soft tissues may follow surgical procedures, ischaemia, or animal or human bites (3). Anaerobic streptococcal myonecrosis is usually associated with a mixed flora, arising from endogenous or 3 exogenous contamination of a wound. The polymicrobial $\mathbb{Q}$ nature of infection is reportedly demonstrated in $76 \%$ of cases (4).

The case we have reported illustrates many of the.? characteristic manifestations of anaerobic streptococcal $\overrightarrow{\vec{F}}$ myonecrosis. We believe, the key to successful treatmento is to recognize the urgency. The presence of gas is $\frac{e}{0}$ thought to be due to anaerobic infection but in fact, $\overline{\bar{c}}$. awaiting bacterial identification could lead to delay in management. Most authors recommend limited surgicalo interventions combined with administrations of antibioticses with rapid clinical improvement after the onset of $\overrightarrow{0}$ appropriate therapy (5-7). In several cases fluid $\overrightarrow{\vec{H}}$ resuscitation and and cardio-respiratory stabilization is essential. If the spread of the infection could not be arrested, repeated inspections and debridement are necessary (8). Hyperbaric oxygenation may have value as? an additive treatment but should not replace surgical $\vec{f}$ debridement, local oxygen therapy with irrigation of the wound with hydrogen peroxide and antibiotic theragy' $(3,9)$.

We conclude, that the invasive nature of this anaerobf을 streptococcal myonecrosis, defined the condition having a potentially rapid lethal course. Therefore, tofe disease required early suspicion, recognition affio appropriate management with both systematic and lo@ treatment, in addition with hyperbaric oxygenation.

\section{REFERENCES}

1. BROOK I. Microbiology of infected pilonidal sinuse; $J$ Clin Pathol 1989; 42: 1140-1142.

2. Brook I, Anderson K D, Controni G, Rodriguezo W J. Aerobic and anaerobic bacteriology of pilonida cyst abscess in children. Am J Dis Child 1980: 134:@ 679-680.

3. JOHNSON C C, FINEGOLD S M. Anaerobic infections in surgery. Probl Gen Surg 1993: 10: 579-594.

4. Freischlag J A, ajalat G, Busuttil R W. Treatment of necrotizing soft tissue infections. The needB for a new approach. Am J Surg 1985: 149: 751-755.

5. Anderson C B, Marr J J, Jaffe B M. Anaerobic streptococcal infectious simulating gas gangrene. $\bar{\Phi}$ Arch Surg 1972; 104: 186-189.

6. Bessman A N, WaGner W. Nonclostridial gas gangrene. Report of 48 cases and review of the literature 3 JAMA 1975; 233: 958-963.

7. BUBRICK M P, HITCHCOCK C R. Necrotizing anorectaB and perineal infections. Surgery 1979; 86: 655-662.

8. Dellinger E P. Severe necrotizing soft-tissue infections. Multiple disease entities requiring a commoro approach. JAMA 1981; 246: 1717-1721.

9. Skiles M S, Covert G K, Fletcher h S. Gas $\stackrel{\sim}{\sigma}$ producing clostridial and nonclostridial infections $\tilde{O}$ Surg Gynecol Obstet 1978; 147: 65-67.

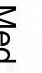

